Blow up points and the Morse indices of solutions to the Liouville equation: inhomogeneous case

Futoshi Takahashi

\begin{tabular}{|c|l|}
\hline Citation & OCAMI Preprint Series \\
\hline Issue Date & 2011 \\
\hline Type & Preprint \\
\hline Textversion & Author \\
\hline
\end{tabular}

From: Osaka City University Advanced Mathematical Institute

http://www.sci.osaka-cu.ac.jp/OCAMI/publication/preprint/preprint.html 


\title{
Blow up points and the Morse indices of solutions to the Liouville equation: inhomogeneous case
}

\author{
Futoshi Takahashi
}

\begin{abstract}
.
Let us consider the Liouville equation

$$
-\Delta u=\lambda V(x) e^{u} \quad \text { in } \Omega, \quad u=0 \quad \text { on } \partial \Omega,
$$

where $\Omega$ is a smooth bounded domain in $\mathbb{R}^{2}, V(x)>0$ is a given function in $C^{1}(\bar{\Omega})$, and $\lambda>0$ is a constant. Let $\left\{u_{n}\right\}$ be an $m$-point blowing up solution sequence for $\lambda=\lambda_{n} \downarrow 0$, in the sense that

$$
\lambda_{n} \int_{\Omega} V(x) e^{u_{n}} d x \rightarrow 8 \pi m \quad \text { as } n \rightarrow \infty
$$

for $m \in \mathbb{N}$. We prove that the number of blow up points $m$ is less than or equal to the Morse index of $u_{n}$ for $n$ sufficiently large. This extends the main result of the recent paper [13] to an inhomogeneous $(V \not \equiv 1)$ case.
\end{abstract}

\section{$\S 1 . \quad$ Introduction}

In this paper we study the Liouville equation

$$
\begin{cases}-\Delta u=\lambda V(x) e^{u} & \text { in } \Omega, \\ u=0 & \text { on } \partial \Omega\end{cases}
$$

where $\Omega$ is a smooth bounded domain in $\mathbb{R}^{2}, V(x)>0$ is a given function in $C^{1}(\bar{\Omega})$, and $\lambda>0$ is a constant.

The purpose of this note is to extend the main result of the recent paper [13], where only $V \equiv 1$ was considered, to the present case.

The Liouville equation appears in several fields of mathematics and physics, and the study of it has a rather long history; see for example, [3], [4], [12], and the references therein.

2000 Mathematics Subject Classification. 35B40, 35J25.

Key words and phrases. Morse index, blow up point, Liouville equation. 
Let $\left\{\lambda_{n}\right\}$ be a sequence of positive numbers with $\lambda_{n} \rightarrow 0$ as $n \rightarrow \infty$. One of the interesting issues of this problem is the study of asymptotic behavior of solutions as $n \rightarrow \infty$. Concerning this, Ma and Wei [10] proved the following fact, which extends the former result by Nagasaki and Suzuki [11] where $V \equiv 1$ was considered.

Theorem 1. (Ma and Wei [10]) For any solution sequence $\left\{u_{n}\right\}$ of (1) for $\lambda=\lambda_{n} \downarrow$, there exists a subsequence (denoted by $u_{n}$ again) such that it holds

$$
\lambda_{n} \int_{\Omega} V(x) e^{u_{n}} d x \rightarrow 8 \pi m, \quad \text { for some } m \in\{0\} \cup \mathbb{N} \cup\{+\infty\},
$$

and according to the cases, the solution sequence $\left\{u_{n}\right\}$ behaves as

(i) uniform convergence to $0:\left\|u_{n}\right\|_{L^{\infty}(\Omega)} \rightarrow 0$, when $m=0$,

(ii) entire blow-up: $u_{n}(x) \rightarrow+\infty$ as $n \rightarrow \infty$ for any $x \in \Omega$ when $m=+\infty$,

(iii) $m$-points blow-up: there exists an m-points set $\mathcal{S}=\left\{a_{1}, \cdots, a_{m}\right\}$, called blow up set, such that each $a_{i}$ is an interior point of $\Omega$, $\left\|u_{n}\right\|_{L^{\infty}(K)}=O(1)$ for any compact set $K \subset \bar{\Omega} \backslash \mathcal{S},\left.u_{n}\right|_{\mathcal{S}} \rightarrow$ $+\infty$, and

$$
u_{n} \rightarrow 8 \pi \sum_{i=1}^{m} G\left(\cdot, a_{i}\right) \quad \text { in } C_{l o c}^{2}(\bar{\Omega} \backslash \mathcal{S})
$$

as $n \rightarrow \infty$ when $m \in \mathbb{N}$. Furthermore, any blow up point $a_{i} \in \mathcal{S}$ must satisfy the condition

$$
\frac{1}{2} \nabla R\left(a_{i}\right)+\sum_{j=1, j \neq i}^{m} \nabla_{x} G\left(a_{i}, a_{j}\right)+\frac{1}{8 \pi} \nabla \log V\left(a_{i}\right)=0
$$

for $i=1,2, \cdots, m$. Here, $G=G(x, y)$ is the Green function of $-\Delta$ under the Dirichlet boundary condition with a pole $y \in$ $\Omega$, and $R(x)=\left[G(x, y)+\frac{1}{2 \pi} \log |x-y|\right]_{y=x}$ denotes the Robin function.

Later, the existence of multiple blowing up solutions with a prescribed blow up set is established; see [6] [7].

Let $i_{M}(u)$ denote the Morse index of a solution $u$ of (1), that is, the number of negative eigenvalues of the linearized operator $L_{u}=-\Delta-$ $\lambda V(x) e^{u}$. acting on $H_{0}^{1}(\Omega)$. In this note, we prove the following, which is an extension of the main theorem in [13] to the inhomogeneous case. 
Theorem 2. Let $\left\{u_{n}\right\}$ be a solution sequence of (1) for $\lambda=\lambda_{n}$ satisfying

$$
\lambda_{n} \int_{\Omega} V(x) e^{u_{n}} d x \rightarrow 8 \pi m
$$

for some $m \in \mathbb{N}$. Then $m \leq i_{M}\left(u_{n}\right)$ for $n$ sufficiently large.

In the homogeneous $(V \equiv 1)$ case [13], we used the fact that $w(x)=$ $(x-a) \cdot \nabla u_{n}(x)+2$ satisfies the equation $-\Delta w=\lambda_{n} e^{u_{n}} w$ (except for the boundary condition) for $a \in \mathbb{R}^{2}$. This is no longer true when $V$ is not a constant, and we need another method. The proof presented here works also for the homogeneous case and the main idea originates from [1].

\section{§. Proof of Theorem 2}

In this section, we prove Theorem 2 along the line of [13].

Let $\left\{u_{n}\right\}$ be a solution sequence to (1) for $\lambda=\lambda_{n}$ with $\lambda_{n} \int_{\Omega} V(x) e^{u_{n}} d x \rightarrow$ $8 \pi m$ for some $m \in \mathbb{N}$. Theorem 1 implies that the existence of the blow up set $\mathcal{S}=\left\{a_{1}, \cdots, a_{m}\right\} \subset \Omega$. Also we have a sufficiently small $\rho>0$ and $m$ sequences of local maximum points $\left\{x_{n}^{i}\right\}$ such that for each $a_{i} \in \mathcal{S}$,

$$
u_{n}\left(x_{n}^{i}\right)=\max _{B_{\rho}\left(x_{n}^{i}\right)} u_{n}(x) \rightarrow \infty, \quad x_{n}^{i} \rightarrow a_{i}(i=1, \cdots, m),
$$

as $n \rightarrow \infty$.

Now we recall the following local pointwise estimate for the blowingup solutions to (1) thanks to YanYan Li [8]: For a fixed $\rho \in(0,1)$, there exists a constant $C>0$ independent of $i=1, \cdots, m$ and $\lambda_{n}>0$ such that

(3) $\left|u_{n}(x)-\log \frac{e^{u_{n}\left(x_{n}^{i}\right)}}{\left(1+\frac{\lambda_{n}}{8} V\left(x_{n}^{i}\right) e^{u_{n}\left(x_{n}^{i}\right)}\left|x-x_{n}^{i}\right|^{2}\right)^{2}}\right| \leq C$ for $x \in B_{\rho}\left(x_{n}^{i}\right)$

holds true.

Here we show a proof for the reader's convenience. Define $v_{n}(x)=$ $u_{n}(x)+\log \lambda_{n}$. Then $v_{n}$ satisfies

$$
-\Delta v_{n}=V(x) e^{v_{n}} \quad \text { in } \Omega, \quad v_{n}=\log \lambda_{n} \quad \text { on } \partial \Omega .
$$

Furthermore, by the assumption $\lambda_{n} \int_{\Omega} V(x) e^{u_{n}} d x \rightarrow 8 \pi m$ and $0<\exists a \leq$ $V(x) \leq \exists b<+\infty$, we see that $\int_{\Omega} e^{v_{n}} d x=O(1)$ as $n \rightarrow \infty$.

Now, we claim that $v_{n}\left(x_{n}^{i}\right) \rightarrow+\infty$ as $n \rightarrow \infty$ for any $i \in\{1, \cdots, m\}$. Indeed, assume the contrary that there exists $i \in\{1, \cdots, m\}$ and a subsequence (denoted by the same symbol) such that 
(i) $v_{n}\left(x_{n}^{i}\right) \rightarrow-\infty$, or

(ii) $v_{n}\left(x_{n}^{i}\right) \rightarrow C$ for some $C \in \mathbb{R}$.

When (i) happens, we see

$$
\int_{B_{\rho}\left(x_{n}^{i}\right)} V(x) e^{v_{n}(x)} d x \leq e^{v_{n}\left(x_{n}^{i}\right)} \int_{B_{\rho}\left(x_{n}^{i}\right)} V(x) d x \rightarrow 0
$$

as $n \rightarrow \infty$. However, this contradicts the fact that

$$
\lim _{n \rightarrow \infty} \int_{B_{\rho}\left(x_{n}^{i}\right)} V(x) e^{v_{n}} d x \geq 8 \pi,
$$

see, for example, Li and Shafrir [9].

Also if (ii) happens, a result of Brezis and Merle ([2]:Theorem 3) implies that $\left\{v_{n}\right\}$ is bounded in $L_{l o c}^{\infty}(\Omega)$. On the other hand, (2) in Theorem 1 implies that $v_{n}=u_{n}+\log \lambda_{n} \rightarrow-\infty$ on any compact set in $\bar{\Omega} \backslash \mathcal{S}$. Thus again we have a contradiction and we have proved the claim.

Once we have the claim, we are in the same situation of Theorem 0.3 in [8] (setting that $\Omega=B_{\rho}\left(x_{n}^{i}\right), 0=x_{n}^{i}$ there). Note that

$$
\max _{\partial B_{\rho}\left(x_{n}^{i}\right)} v_{n}(x)-\min _{\partial B_{\rho}\left(x_{n}^{i}\right)} v_{n}(x)=\max _{\partial B_{\rho}\left(x_{n}^{i}\right)} u_{n}(x)-\min _{\partial B_{\rho}\left(x_{n}^{i}\right)} u_{n}(x)=O(1)
$$

as $n \rightarrow \infty$. Thus by Theorem 0.3 in [8], we have

$$
\left|v_{n}(x)-\log \frac{e^{v_{n}\left(x_{n}^{i}\right)}}{\left(1+\frac{\lambda_{n}}{8} V\left(x_{n}^{i}\right) e^{v_{n}\left(x_{n}^{i}\right)}\left|x-x_{n}^{i}\right|^{2}\right)^{2}}\right| \leq C \quad \text { for } x \in B_{\rho}\left(x_{n}^{i}\right),
$$

which is equivalent to (3).

Now, let us define

$$
\begin{aligned}
& \left(\delta_{n}^{i}\right)^{2} \lambda_{n} e^{u_{n}\left(x_{n}^{i}\right)}=1, \\
& \tilde{u}_{n}^{i}(y)=u_{n}\left(\delta_{n}^{i} y+x_{n}^{i}\right)-u_{n}\left(x_{n}^{i}\right), \quad y \in B_{\rho / \delta_{n}^{i}}(0)
\end{aligned}
$$

for $i \in\{1, \cdots, m\}$. By the above pointwise estimate, we easily see that $\delta_{n}^{i}=o(1)$ as $n \rightarrow \infty$. The scaled function $\tilde{u}_{n}^{i}$ satisfies

$$
\left\{\begin{array}{l}
-\Delta \tilde{u}_{n}^{i}=V\left(\delta_{n}^{i} y+x_{n}^{i}\right) e^{\tilde{u}_{n}^{i}} \quad \text { in } B_{\rho / \delta_{n}^{i}}(0), \\
\tilde{u}_{n}^{i}(0)=0, \tilde{u}_{n}^{i}(x) \leq 0, \quad \forall x \in B_{\rho / \delta_{n}^{i}}(0), \\
\int_{B_{\rho / \delta_{n}^{i}}(0)} V\left(\delta_{n}^{i} y+x_{n}^{i}\right) e^{\tilde{u}_{n}^{i}} d y=O(1), \quad(n \rightarrow \infty) .
\end{array}\right.
$$


Moreover, by an argument in [13], we obtain

(4) $\quad \tilde{u}_{n}^{i} \rightarrow U^{i}(y)=-2 \log \left(1+\frac{V\left(a_{i}\right)}{8}|y|^{2}\right) \quad$ for $i=1, \cdots, m$

in $C_{l o c}^{1}\left(\mathbb{R}^{2}\right)$ as $n \rightarrow \infty$, where $U^{i}$ is a unique ([5]) solution of

$$
\left\{\begin{array}{l}
-\Delta U^{i}=V\left(a_{i}\right) e^{U^{i}} \quad \text { in } \mathbb{R}^{2} \\
U^{i}(0)=0, U^{i}(y) \leq 0, \quad \forall y \in \mathbb{R}^{2} \\
\int_{\mathbb{R}^{2}} e^{U^{i}} d y<+\infty
\end{array}\right.
$$

Now, we define two elliptic operators

$$
\begin{aligned}
& L_{n}:=-\Delta_{x}-\lambda_{n} V(x) e^{u_{n}(x)} \cdot: H_{0}^{1}(\Omega) \rightarrow H^{-1}(\Omega), \\
& \tilde{L}_{n}^{i}:=-\Delta_{y}-V\left(\delta_{n}^{i} y+x_{n}^{i}\right) e^{\tilde{u}_{n}^{i}(y)} \cdot: H_{0}^{1}\left(B_{\rho / \delta_{n}^{i}}(0)\right) \rightarrow H^{-1}\left(B_{\rho / \delta_{n}^{i}}(0)\right) .
\end{aligned}
$$

These two operators are related to each other by the formula

$$
\left.\left(\delta_{n}^{i}\right)^{2} L_{n}\right|_{u_{n}(x)=\tilde{u}_{n}^{i}(y)+u_{n}\left(x_{n}^{i}\right)}=\tilde{L}_{n}^{i}
$$

where $x=\delta_{n}^{i} y+x_{n}^{i}$ for $x \in B_{\rho}\left(x_{n}^{i}\right)$ and $y \in B_{\rho / \delta_{n}^{i}}(0)$. Also for a domain $D \subset B_{\rho}\left(x_{n}^{i}\right)$, we have

$$
\left(\delta_{n}^{i}\right)^{2} \lambda_{j}\left(L_{n}, D\right)=\lambda_{j}\left(\tilde{L}_{n}^{i}, D_{n}^{i}\right), \quad D_{n}^{i}=\frac{D-x_{n}^{i}}{\delta_{n}^{i}}
$$

where $\lambda_{j}\left(L_{n}, D\right), \lambda_{j}\left(\tilde{L}_{n}^{i}, D_{n}^{i}\right)(j \in \mathbb{N})$ denote the $j$-th eigenvalue of elliptic operators $L_{n}, \tilde{L}_{n}^{i}$ acting on $H_{0}^{1}(D), H_{0}^{1}\left(D_{n}^{i}\right)$ respectively.

We show the following.

Lemma 2.1. There exists $R>0$ such that $\lambda_{1}\left(L_{n}, B_{\delta_{n}^{i} R}\left(x_{n}^{i}\right)\right)<0$ for $n$ large and for any $i \in\{1, \cdots, m\}$. Furthermore, these $m$ balls are disjoint for $n$ large.

Proof. For $R>0$, we define

$$
w_{R}(y)=2 \log \frac{8+R^{2}}{8+|y|^{2}} .
$$

Since $w_{R}=0$ on $\partial B_{R}(0)$, we see $w_{R} \in H_{0}^{1}\left(B_{R}(0)\right)$. 
We will prove that $\left(\tilde{L}_{n}^{i} w_{R}, w_{R}\right)_{L^{2}\left(B_{R}\right)}<0$ for $R>0$ sufficiently large and $B_{R}(0) \subset B_{\rho / \delta_{n}^{i}}(0)$. Indeed,

$$
\begin{aligned}
\left(\tilde{L}_{n}^{i} w_{R}, w_{R}\right)_{L^{2}\left(B_{R}\right)} & =\int_{B_{R}(0)}\left|\nabla w_{R}\right|^{2} d y-\int_{B_{R}(0)} V\left(\delta_{n}^{i} y+x_{n}^{i}\right) e^{\tilde{u}_{n}(y)} w_{R}^{2}(y) d y \\
& =: I_{1}-I_{2} .
\end{aligned}
$$

We observe that

$I_{1}=\int_{B_{R}(0)} \frac{16|y|^{2}}{\left(8+|y|^{2}\right)^{2}} d y=2 \pi \int_{0}^{R} \frac{16 r^{2}}{\left(8+r^{2}\right)^{2}} r d r=32 \pi\left[\log R+o_{R}(1)\right]$,

where $o_{R}(1) \rightarrow 0$ as $R \rightarrow \infty$. On the other hand, we have

$$
\begin{aligned}
I_{2} & =\int_{B_{R}(0)} V\left(\delta_{n}^{i} y+x_{n}^{i}\right) e^{\tilde{u}_{n}(y)} w_{R}^{2}(y) d y \\
& =V\left(a_{i}\right) \int_{B_{R}(0)} \frac{1}{\left(1+\frac{V\left(a_{i}\right)}{8}|y|^{2}\right)^{2}}\left\{\log \frac{8+R^{2}}{8+|y|^{2}}\right\}^{2} d y+o_{n}(1) \\
& =2 \pi V\left(a_{i}\right) \int_{0}^{R} \frac{r}{\left(1+\frac{V\left(a_{i}\right)}{8} r^{2}\right)^{2}}\left\{\log \left(8+R^{2}\right)-\log \left(8+r^{2}\right)\right\}^{2} d r+o_{n}(1) \\
& =2 \pi V\left(a_{i}\right) \cdot 8^{2}\left\{\log \left(8+R^{2}\right)\right\}^{2}\left[\frac{1}{16 V\left(a_{i}\right)}+o_{R}(1)\right]+o_{n}(1) \\
& =32 \pi(\log R)^{2}\left[1+o_{R}(1)\right]+o_{n}(1),
\end{aligned}
$$

where we have used (4) and

$$
\int_{0}^{R} \frac{r}{\left(8+c r^{2}\right)^{2}} d r=\int_{0}^{\infty} \frac{r}{\left(8+c r^{2}\right)^{2}} d r+o_{R}(1)=\frac{1}{16 c}+o_{R}(1)
$$

for $c>0$. Thus we obtain

$$
\left(\tilde{L}_{n}^{i} w_{R}, w_{R}\right)_{L^{2}\left(B_{R}\right)}=I_{1}-I_{2}=-32 \pi(\log R)^{2}\left[1+o_{R}(1)\right]<0
$$

by taking $n$ sufficiently large first, and then $R>0$ large such that $B_{R}(0) \subset B_{\rho / \delta_{n}^{i}}(0)$. This implies that the first eigenvalue of the operator $\tilde{L}_{n}^{i}$ on $B_{R}$ is negative: $\lambda_{1}\left(\tilde{L}_{n}^{i}, B_{R}\right)<0$. By this and the scaling formula (5) proves the first half part of the Lemma.

The fact that these balls $B_{\delta_{n}^{i} R}\left(x_{n}^{i}\right)$ are disjoint follows from the strict concavity of the limit functions $U^{i}(y)=-2 \log \left(1+\frac{V\left(a_{i}\right)}{8}|y|^{2}\right)$; see [13]. 
By Lemma 2.1, we have $m$ open balls $B^{1}, \cdots, B^{m}, B^{i}=B_{\delta_{n}^{i} R}\left(x_{n}^{i}\right)$, which are disjoint, and

$$
\lambda_{1}\left(L_{n}, B^{i}\right)<0 \quad \text { for } i=1, \cdots, m .
$$

On the other hand, it is easy to see that

$$
\lambda_{m}\left(L_{n}, \Omega\right) \leq \sum_{i=1}^{m} \lambda_{1}\left(L_{n}, B^{i}\right)
$$

holds; see for example, [13]. Combining these inequalities, we have $\lambda_{m}\left(L_{n}, \Omega\right)<0$. Therefore by the definition of the Morse index of $u_{n}$, we have $m \leq i_{M}\left(u_{n}\right)$. This proves Theorem 2 .

Acknowledgments. Part of this work was supported by JSPS Grantin-Aid for Scientific Research (B), No. 23340038.

\section{References}

[1] M. Ben Ayed, K. El Mehdi, and M. Grossi: Asymptotic behavior of least energy solutions of a biharmonic equation in dimension four, Indiana Univ. Math. J. 55 (2006) 1723-1750.

[2] H. Brezis, and F. Merle: Uniform estimates and blow-up behavior for solutions of $-\Delta u=V(x) e^{u}$ in two dimensions, Comm. Partial Differential Equations 16 (1991) 1223-1253.

[3] E. Caglioti, P.L. Lions, C. Marchioro, and M. Pulvirenti: A special class of stationary flows for two-dimensional Euler equations: a statistical mechanics description, I \& II. Comm. Math. Phys. 143 (1992) 501-525 \& 174 (1995) 229-260.

[4] S.Y.A. Chang, and P.C. Yang: Prescribing Gaussian curvature on $S^{2}$, Acta Math. 159 (1987) 215-259.

[5] E. Chen, and C. Li: Classification of solutions of some nonlinear elliptic equations, Duke Math. J. 63(3) (1991) 615-622.

[ 6 ] M. del Pino, M. Kowalczyk and M. Musso: Singular limits in Liouville-type equations, Calc. Var. and Partial Differential Equations 24 (2005) 47-81.

[ 7 ] P. Esposito, M. Grossi and A. Pistoia: On the existence of blowing-up solutions for a mean field equation, Ann. I. H. Poincaré 22 (2005) 227-257.

[ 8 ] Y. Y. Li: Harnack type inequality: the method of moving planes, Commun. Math. Phys. 200 (1999) 421-444.

[9] Y. Y. Li, and I. Shafrir: Blow-up analysis for solutions of $-\Delta u=V e^{u}$ in dimension two, Indiana Univ. Math. J. 43 (1994) 1255-1270.

[10] L. Ma, and J. Wei: Convergence for a Liouville equation, Comment. Math. Helv. 76 (2001) 506-514. 
[11] K. Nagasaki, and T. Suzuki: Asymptotic analysis for two-dimensional elliptic eigenvalue problems with exponentially dominated nonlinearities, Asymptotic Anal. 3 (1990) 173-188.

[12] M. Struwe, and G. Tarantello: On multivortex solutions in Chern-Simons gauge theories, Boll. Unione Mat. Ital. Sez. B. 8 (1998) 109-121.

[13] F. Takahashi: Blow up points and the Morse indices of solutions to the Liouville equation in two-dimension, to appear in Advances in Nonlinear Studies

Department of Mathematics, Osaka City University

83 Osaka City University Advanced Mathematical Institute

Sumiyoshi-ku, Osaka, 558-8585, Japan

E-mail address: futoshi@sci.osaka-cu.ac.jp 